\title{
Can Iterative Reconstruction Improve Imaging Quality for Lower Radiation CT Perfusion? Initial Experience
}

\author{
C.J. Lin, T.H. Wu, C.H. Lin, S.C. Hung, C.F. Chiu, M.-J. Liu, M.M.H. Teng, F.C. Chang, W.Y. Guo, and C.Y. Chang
}

\begin{abstract}
BACKGROUND AND PURPOSE: Initial results using IR for CT of the head showed satisfactory subjective and objective imaging quality with a $20-40 \%$ radiation dose reduction. The aim of our study was to compare the influence of IR and FBP algorithms on perfusion parameters at standard and lowered doses of CTP.
\end{abstract}

MATERIALS AND METHODS: Forty patients with unilateral carotid stenosis post-carotid stent placement referred for follow-up CTP were divided into 2 groups (tube currents were $100 \mathrm{mAs}$ in group A and $80 \mathrm{mAs}$ in group B). Datasets were reconstructed with IR and FBP algorithms; and SNRs of gray matter, white matter, and arterial and venous ROIs were compared. CBF, CBV, and MTT means and SNRs were evaluated by using linear regression, and qualitative imaging scores were compared across the 2 algorithms.

RESULTS: The mean effective radiation dose of group B ( $2.06 \mathrm{mSv}$ ) was approximately $20 \%$ lower than that of group A ( $2.56 \mathrm{mSv})$. SNRs for ROIs in the dynamic contrast-enhanced images were significantly higher than those for the FBP images. Correlations of the SNRs for CBF, CBV, and MTT across the 2 algorithms were moderate $\left(R^{2}=0.46,0.23\right.$, and 0.44 , respectively). ROls in gray matter rather than the IR algorithm predicted increasing SNRs in all CBF, CBV, and MTT maps. Two cases of significant restenosis were confirmed in both algorithms. $\mathrm{CBV}, \mathrm{CBF}$, and MTT imaging scores did not differ significantly across algorithms or groups.

CONCLUSIONS: Lower dose CTP (20\% below normal dose) without IR can effectively identify oligemic tissue in poststenting follow-up. IR does not alter the absolute values or increase the SNRs of perfusion parameters. Other methods should be attempted to improve SNRs in settings with low tube currents.

ABBREVIATIONS: $\mathrm{FBP}=$ filtered back-projection; $I R=$ iterative reconstruction; $T$ max $=$ time point of maximal enhancement; VOF $=$ venous output function

in $\mathrm{R}$ has been shown to be an effective reconstruction method for improving imaging quality at low radiation doses in CT of the chest and abdomen. ${ }^{1-3}$ Current IR techniques attempt to formulate image reconstruction as an optimization problem in both signal space and imaging space. The noisiest measurements are given low weight in the iterative process by an intensive computerized process and, therefore, contribute very little to the final image. ${ }^{4}$ Initial results by using IR for CT of the head showed satisfactory subjective and objective imaging quality with a $20 \%-$ $40 \%$ radiation dose reduction. ${ }^{3,5,6}$ An integrated IR algorithm

Received August 31, 2012; accepted after revision October 10.

From the Department of Radiology (C.J.L., S.C.H., C.F.C., M.-J.L., M.M.H.T., F.C.C., W.Y.G., C.Y.C.), Taipei Veterans General Hospital, Taipei, Taiwan; School of Medicine (C.J.L., S.C.H., C.F.C., M.-J.L., M.M.H.T., F.C.C., W.Y.G., C.Y.C.) and Department of Biomedical Imaging and Radiological Sciences (T.H.W.), National Yang-Ming University, Taipei, Taiwan; and Graduate Institute of Epidemiology and Preventive Medicine (C.H.L.), National Taiwan University, Taipei, Taiwan.

Please address correspondence to Sheng Che Hung, MD, Department of Radiology, Taipei Veterans General Hospital, No. 201, Sec. II, Shipai Rd, Taipei, 11217 Taiwan; e-mail: hsz829@gmail.com

http://dx.doi.org/10.3174/ajnr.A3436
iDose $^{4}$ (Philips Healthcare, Best, the Netherlands) has recently been developed, which consists of the following 2 de-noising components: an iterative maximum likelihood-type sinogram restoration method based on Poisson noise distribution and a local structure model fitting on image data that iteratively decreases the uncorrelated noise. In clinical practice, the users can adjust the image noise by inputting a parameter called "iDose level."7

In 2009, the FDA raised concern about the radiation dose accompanying brain CTP in daily neurovascular examinations. ${ }^{8}$ CTP has been widely used to evaluate hemodynamic changes in the brains of patients with stenotic-occlusive diseases and those with subarachnoid hemorrhage. ${ }^{9,10}$ In combination with CT angiography, it serves as an excellent triage tool for patients with acute ischemic insults who may benefit from thrombolytic therapy within and beyond the therapeutic time window. ${ }^{11,12}$ With quick acquisition processes and lower radiation doses, CTP serves as a potential peritherapeutic hemodynamic monitoring tool for patients with neurovascular disorders. 
There are 2 approaches to lowering radiation doses during CTP. Prolonged scanning intervals can reduce the dose by $25 \%-$ $58 \%$, depending on the frequency of sampling on the time-attenuation curve. For a fixed sampling frequency, the radiation dose of diagnostic-qualitative MTT mapping can be decreased to $58.3 \% .{ }^{13,14}$ For an alternative sampling frequency in the arterial and venous phases, the maximum interval to provide an absolute CBF value is 1.5 seconds in the arterial phase and 3 seconds in the venous phase, resulting in a dose reduction of approximately $38 \% .{ }^{14-16}$ Keeping the kilovolt(peak) at the level of 80 is recommended to provide the best contrast-to-noise ratio for CTP. ${ }^{17}$ These temporal variables dominate the SNRs in CTP. ${ }^{16}$ Lowering the tube current is the second method used to decrease the radiation dose. Its influence on hemodynamic parameters such as $\mathrm{CBV}, \mathrm{CBF}$, and MTT is not well-understood. The aim of this study was to explore the effects of IR on the imaging quality of primary enhanced images and color maps of hemodynamic parameters in CTP in a setting of lower tube currents.

\section{MATERIALS AND METHODS \\ Patient Selection}

The local institutional review board approved the study. Informed consent was obtained before CTP. From February to April 2012, forty-two patients with unilateral carotid stenosis who had undergone stent placement from 6 to 12 months before the start of the study were referred for regular follow-up of CTA and CTP and were prospectively enrolled in our study. One patient with poor heart function and 1 with poor renal function were excluded. All patients were randomly assigned to 1 of 2 subgroups. Group A underwent CTP with a standard radiation dose at a tube kilovolt(peak) of 80 and a tube current of 100 mAs. Group B underwent CTP with a reduced radiation dose at a tube kilovolt(peak) of 80 and a tube current of 80 mAs.

\section{CTP Acquisition and Dataset Reconstruction}

All examinations were performed on the Brilliance iCT scanner (Philips Healthcare). The CT perfusion protocol was as follows: FOV , $20 \mathrm{~cm}$; matrix, $512 \times 512$; scan range, $8 \mathrm{~cm}$ from the sella floor to the high vertex. Each scan lasted 60 seconds with a fixed temporal resolution of 1.5 seconds in both the arterial and venous phases. A total of $40 \mathrm{~mL}$ of contrast (iopromide, Ultravist 370; Schering, Berlin, Germany) was injected via the right antecubital vein at a rate of $5 \mathrm{~mL} / \mathrm{s}$. The scanning time started 5 seconds after the initiation of contrast. Besides the regular images reconstructed with FBP, the original datasets were programmed to automatically reconstruct another set of source images by IR directly from the CT console. In general, the optimal level (n) of iDose ${ }^{4}$ used to compensate for the reduced SNR of the reconstructed imaging is according to the reduced radiation $([10 n+10] \%)$. However, this does not necessarily imply that the same percentage of IR in combination with FBP was introduced. For example, to achieve the equivalent SNR as standard CTP, iDose level 1 should be used to compensate for a deteriorated SNR when the effective dose is decreased by $20 \%$. The exact IR percentage is not revealed by the program. Nevertheless, in our study, we used iDose ${ }^{4}$ level 5 for 2 reasons: First, the initial dose-reduction escalation was set as low as the tube current, equal to $40 \mathrm{~mA}$ (the optimal iDose $\mathrm{e}^{4}$ level

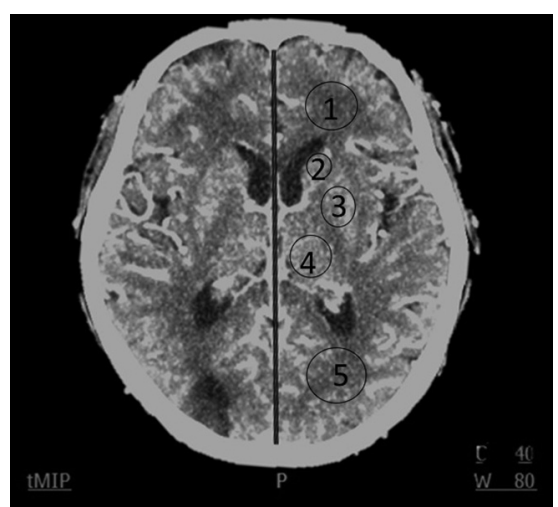

FIG 1. The imaging plane for 5 ROls: frontal white matter (ROII), caudate nucleus (ROI2), lentiform nucleus (ROI3), thalamus (ROI4), and occipital white matter (ROI5).

is 5). However, the first several cases in this group showed unacceptable imaging quality and thus were prematurely terminated. Second, because the main purpose of our study was to determine the effect of IR on the imaging quality of the hemodynamic parameters, we universally used the highest level of iDose ${ }^{4}$ to maximize the effect of IR and facilitate comparison among all subgroups.

\section{Imaging Postprocessing}

The 2 datasets were postprocessed on the workstation (Aquarius, Version 4.4.7; TeraRecon, Foster City, California) for brain perfusion analysis by using a deconvolution method to obtain color maps of the CBV, CBF, and MTT. The arterial region of interest for the arterial input function was placed at the second segment of the anterior cerebral artery, and the venous region of interest for the VOF was placed within the superior sagittal sinus at the same plane. To minimize the selection bias for the arterial input function and VOF between 2 reconstruction algorithms, the determination of arterial and venous ROIs was manually duplicated by side-by-side comparison and set to include the whole cross-section of intended vessels.

For analysis of imaging quality of axial 5-mm dynamic contrast-enhanced images, 5 ROIs at the level where the ventricles and the basal ganglia can be seen optimally at Tmax were manually chosen bilaterally (Fig 1). These 5 ROIs were frontal white matter, occipital white matter, the head of the caudate nucleus, the lentiform nucleus, and the thalamus. We excluded ROIs located in the area of encephalomalacia and postischemic parenchymal changes. The arterial and venous regions of interest at their respective Tmax time points were also included for analysis. Five corresponding ROIs in the color maps of CBV, CBF, and MTT were chosen for intergroup comparison (Fig $2 A-C$ ). The sizes of ROIs in the brain parenchyma were created equally in both algorithms and set to $25-40 \mathrm{~mm}^{2}$. The SNR of each region of interest was defined as its mean divided by its SD.

\section{Quantitative and Qualitative Analysis}

For quantitative analysis, the mean values in each region of interest in the axial maximal enhanced image were compared between the 2 reconstruction algorithms. The correlations of means and SNRs of individual ROIs between the 2 reconstruction algorithms 

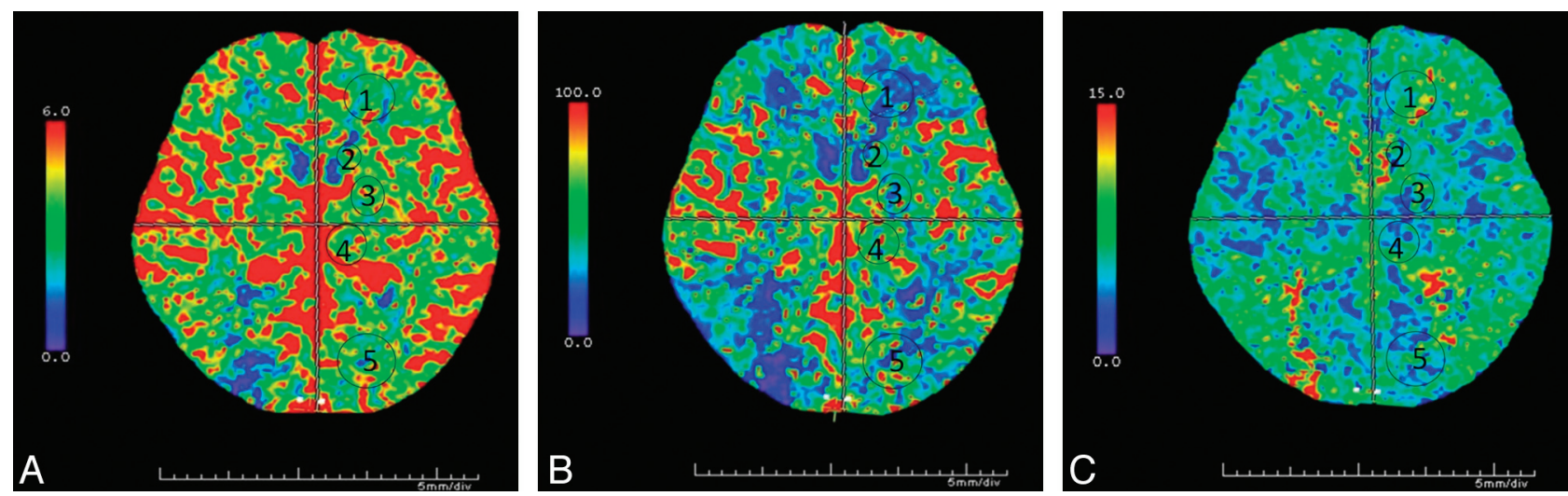

FIG 2. CBV $(A), C B F(B)$, and $M T T(C)$ of lower dose CTP (tube current is $80 \mathrm{mAs}$, group $B$ ) in a 61-year-old man, 6 months after receiving a right carotid artery stent.

Table 1: Patient characteristics and radiation dose in CT protocols with standard and low tube currents

\begin{tabular}{lccc}
\hline Subject Characteristic & $\begin{array}{c}\text { Group A } \\
\text { (Tube Current = 100 mAs) }\end{array}$ & $\begin{array}{c}\text { Group B } \\
\text { (Tube Current = 80 mAs) }\end{array}$ & $\begin{array}{c}\boldsymbol{P} \\
\text { Value }\end{array}$ \\
\hline Age & 74.9 & 74.2 & .48 \\
Sex (M/F) & $14 / 6$ & $14 / 6$ & 1 \\
Tmax (second) & $12.5+2.34$ & $12.3+2.83$ & .53 \\
Maximium HU of A2 ${ }^{\mathrm{a}}$ & 159.84 & 177.6 & .13 \\
CTDI (mGy) & $153.56+0.03$ & $128.17+0.03$ & $<.001$ \\
DLP (mGy/cm) & $1229+0.26$ & $985+0.6$ & $<.001$ \\
Effective dose (mSv) & $2.56 \mathrm{mSv}$ & $2.06 \mathrm{mSv}$ & $<.001$ \\
\hline
\end{tabular}

Note:- Tmax indicates the time point of maximal enhancement of anterior cerebral artery; DLP, dose-length product. ${ }^{\mathrm{a}} \mathrm{A} 2$ indicates second portion of anterior cerebral artery.

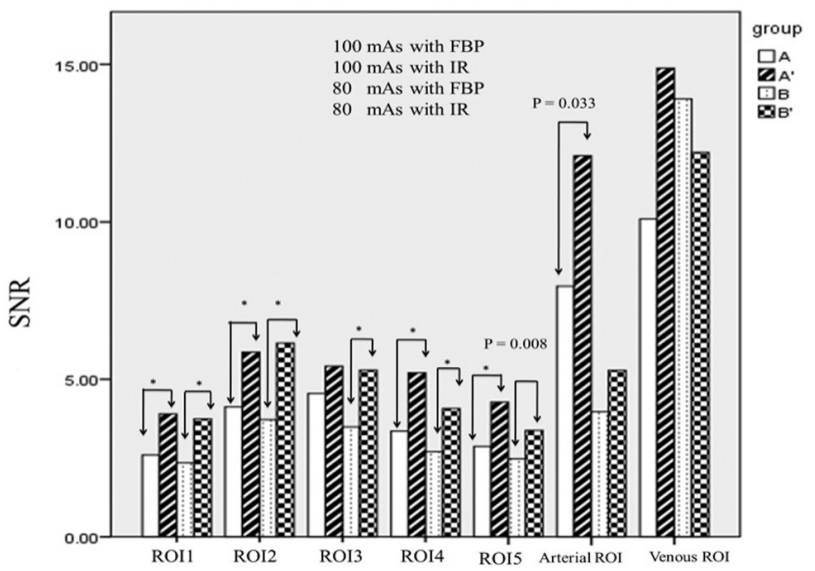

FIG 3. The mean SNR of individual ROls in axial-enhanced imaging at Tmax reconstructed by IR and FBP in groups $A$ and $B$ (group A: 100 mAs with FBP; group A: 100 mAs with IR; group B: 80 mAs with FBP; group B: $80 \mathrm{mAs}$ with IR. Asterisk indicates $P$ value $<.001$ ).

for the 3 perfusion maps (CBV, CBF, and MTT) were also compared. We used the general linear model to evaluate the SNRs. For predictor variables, we used tube current (100 and $80 \mathrm{mAs}$ ), the 2 algorithms, the ROIs in white and gray matter, and the peak arterial and venous enhancement. Previous literature indicated that the latter 2 could influence the SNR of perfusion maps; thus, they were also enrolled in analysis. ${ }^{18}$ For the qualitative assessment, we modified the method proposed by Abels et al, ${ }^{13}$ and rated the color-coded perfusion maps (CBV, CBF, and MTT) on a 3-point scale ( $0=$ poor, $1=$ fair, and $2=$ adequate) for 3 features: graywhite differentiation, homogeneity, and severity of artifacts. The scores for the 3 features were added, resulting in a maximum score of 6 .

Two neuroradiologists with 5 and 3 years' experience interpreted the perfusion imaging. They were not aware of the reconstruction algorithms used or the subgroups of the perfusion. Statistical analysis was conducted with the Statistical Package for the Social Sciences software, Version 16.0 (SPSS, Chicago, Illinois). We used paired $t$ tests to compare differences in SNRs in enhanced imaging between 2 algorithms and 1-sample $t$ tests to compare the differences in imaging scores for the perfusion maps between subgroups. Pearson correlation coefficients were used to compare mean values and SNRs for each region of interest in the perfusion maps for the 2 reconstructive algorithms.

\section{RESULTS}

\section{Patient Demographics, Radiation Dose, and Time-Attenuation Curve Analysis}

There were no significant differences in patient age, sex, Tmax, or peak enhancement of arterial and venous ROIs between the 2 groups (Table 1). The CTDI and effective dose in group B were significantly lower than those in group A. There were 2 significant restenoses identified by CT angiography and CTP and confirmed by digital subtraction angiography.

\section{Imaging Quality of Dynamic Enhanced Images and CTP Color Maps}

For both group A and B, SNRs of all intraparenchymal ROIs reconstructed by IR were significantly superior to those reconstructed by FBP, except for the lentiform nucleus ROI3 in group A (Fig 3). Arterial and venous ROIs reconstructed by IR demonstrated superior SNRs compared with those reconstructed by FBP, but only arterial ROIs in group A showed significant differences $(P=.03)$. With a reduced tube current of $80 \mathrm{mAs}$, the SNRs of all ROIs within the brain parenchyma reconstructed by IR were still significantly higher than those obtained at a tube current of $100 \mathrm{mAs}$ reconstructed by FBP. For perfusion color-map analysis, the mean value correlations for CBF, CBV, and MTT across the 2 reconstruction algorithms all demonstrated good correlations 

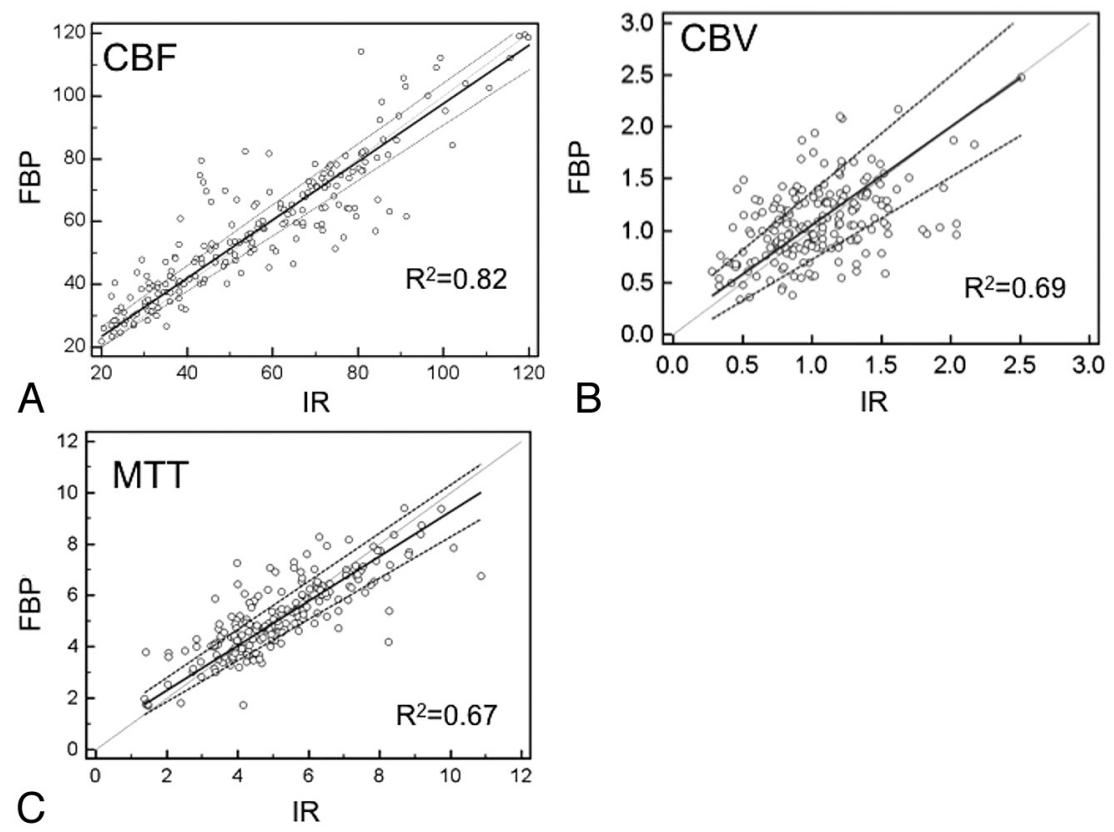

FIG 4. The mean values of individual ROIs in $C B V(A), C B F(B)$, and $M T T(C)$ reconstructed by IR and FBP ( $x$-axis: mean value of IR; $y$-axis: mean value of FBP).
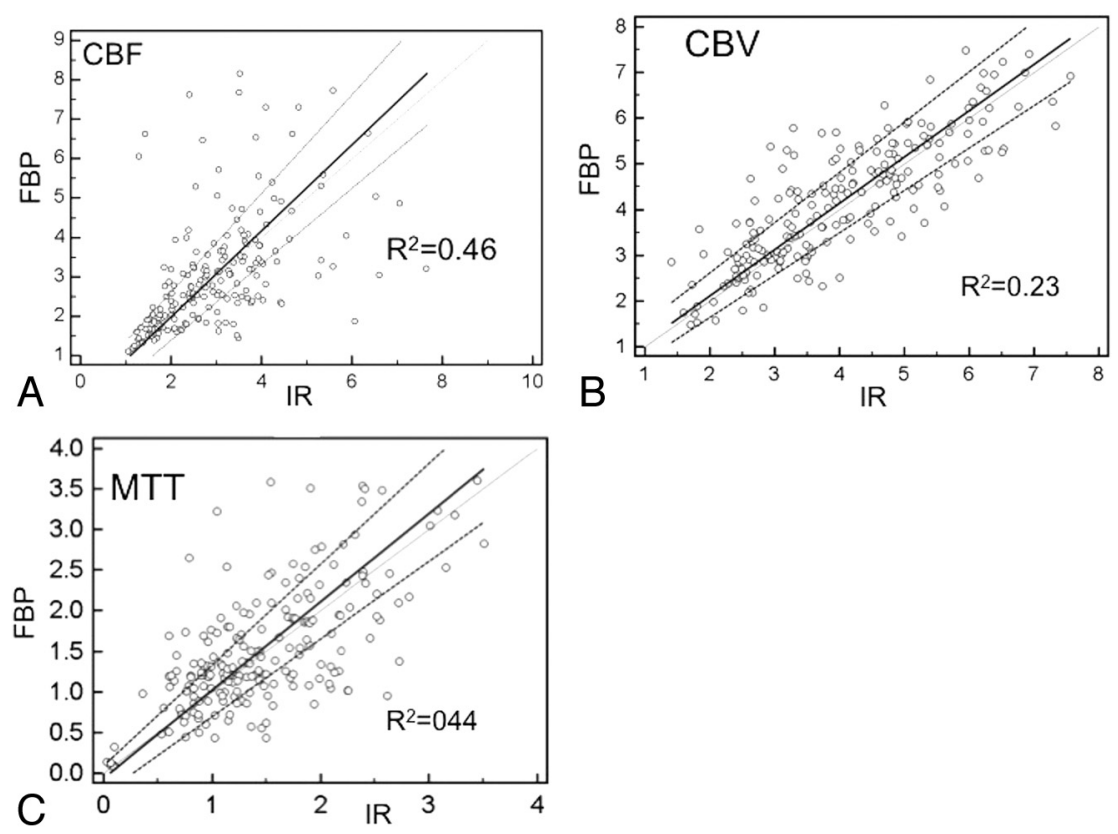

FIG 5. The correlation $S N R$ of individual regions of interest in $C B V(A), C B F(B)$, and $M T T(C)$ reconstructed by IR and FBP ( $x$-axis: SNR of IR; $y$-axis: SNR of FBP).

$\left(R^{2}=0.82,0.69\right.$, and 0.67 , respectively) (Fig 4). The SNR correlations for $\mathrm{CBF}, \mathrm{CBV}$, and MTT across the 2 reconstruction algorithms were moderate $\left(R^{2}=0.46,0.23\right.$, and 0.44 , respectively), regardless of the milliampere-second used or locations (Fig 5).

Results from the general linear regression model showed that the gray matter region of interest was the only independent variable to enhance SNRs in the CBF and CBV maps. For the MTT maps, region of interest in the gray matter, tube current of 100 $\mathrm{mAs}$, and arterial and venous peak enhancement were all found to enhance the SNRs. However, there were no differences in any of the 3 maps in SNR between IR and FBP (Table 2). For color-map interpretations, the $\kappa$ value between the 2 readers was 0.73 . The CBF imaging quality score was nonsignificantly lower in group A compared with group B, regardless of the reconstructive algorithm. For CBV and MTT, there was no difference in subjective imaging quality between standard and lower dose CTP, regardless of the reconstructive algorithms (Table 3).

\section{DISCUSSION}

Normalization of hemodynamic characteristics between 2 groups is challenging in small patient populations. To the best of our knowledge, no commercial interface currently provides a simple variable to represent the shape of the contrast bolus passage (ie, time-attenuation curve). We used the Tmax and maximum Hounsfield units of the arterial region of interest as 2 surrogates to describe the time-attenuation curve with a fixed injected contrast volume and rate. ${ }^{19,20} \mathrm{~Pa}$ tients with poorer heart function demonstrated more prolonged Tmax and lower Hounsfield unit values in the arterial ROIs. In our study, there were no differences in Tmax and Hounsfield units between the 2 groups, which might have indicated that hemodynamics could affect subsequent analysis. Finally, we applied deconvolution methods, which, unlike the maximum slope method, are insensitive to heart function, ${ }^{21,22}$ to acquire the perfusion parameters.

All ROIs in both gray and white matter in axial dynamic contrast-enhanced images demonstrated better SNRs when reconstructed by IR than by FBP, except those in the lentiform nucleus in group A. Our explanation is that the enhanced lenticulostriate arteries within the selected ROIs increased the SD in the comparison of means and thereafter resulted in a trend of better SNR, which nonetheless failed to achieve significance. During the deconvolution algorithm, the signal intensity reflecting the concentration of contrast in the ROIs was the dependent variable in the formula, which theoretically could change the values of $\mathrm{CBV}$ and $\mathrm{CBF} .{ }^{23}$ Our study demonstrated that the changes were not significant and showed good correlation between the 2 algorithms. The differences were even smaller than interobserver and intraobserver variabilities reported in the literature. ${ }^{24,25}$

Earlier research has shown that the SNRs of the perfusion maps can be affected by the placement of arterial and venous 
Table 2: General linear regression of SNR in CBV, CBF, and MTT values among different tube currents, locations, reconstructive algorithms, and peak arterial and venous enhancement

\begin{tabular}{|c|c|c|c|c|c|c|}
\hline & \multicolumn{2}{|c|}{ SNR of CBF } & \multicolumn{2}{|c|}{ SNR of CBV } & \multicolumn{2}{|c|}{ SNR of MTT } \\
\hline & $\begin{array}{l}\text { Standardized } \\
\text { Estimate }\end{array}$ & $P$ Value & $\begin{array}{l}\text { Standardized } \\
\text { Estimate }\end{array}$ & $P$ Value & $\begin{array}{l}\text { Standardized } \\
\text { Estimate }\end{array}$ & $P$ Value \\
\hline $80 \mathrm{mAs}^{\mathrm{a}}$ & 0.043 & .412 & 0.016 & .747 & -0.121 & $.037^{\mathrm{d}}$ \\
\hline IR reconstruction ${ }^{\mathrm{b}}$ & -0.008 & .872 & 0.002 & .655 & 0.007 & .887 \\
\hline Gray matter ${ }^{c}$ & 0.443 & $<.001^{\mathrm{d}}$ & 0.515 & $<.001^{\mathrm{d}}$ & -0.203 & $<.001^{\mathrm{d}}$ \\
\hline Peak enhancement of arterial ROI & 0.055 & .286 & 0.064 & .195 & -.178 & $.001^{\mathrm{d}}$ \\
\hline Peak enhancement of venous ROI & 0.017 & .754 & 0.043 & .408 & -0.163 & $.005^{\mathrm{d}}$ \\
\hline
\end{tabular}

a The control group has a tube current of $100 \mathrm{mAs}$.

b The control group is processed by FBP.

' The control group is ROI white matter, including ROI1 and ROI5; the experimental group is ROI gray matter including ROI2, ROI3, and ROI4

d Statistically significant $(P<.05)$.

Table 3: Mean imaging score of $\mathrm{CBV}, \mathrm{CBF}$, and MTT reconstructed by $I R$ and $F B P$ in groups $A$ and $B$

\begin{tabular}{lccc}
\hline & CBV Score & CBF Score & MTT Score \\
\hline IR & & & \\
$\quad$ Group A (100 mAs) & $5.5 \pm 1.7$ & $4.2+2.0$ & $3.5 \pm 1.4$ \\
$\quad$ Group B (80 mAs) & $5.5 \pm 1.9$ & $4.1 \pm 2.1$ & $3.5 \pm 1.5$ \\
FBP & & & \\
$\quad$ Group A (100 mAs) & $5.3 \pm 1.8$ & $3.6 \pm 2.1$ & $3.4 \pm 1.5$ \\
$\quad$ Group B (80 mAs) & $5.2 \pm 1.9$ & $3.6 \pm 2.0$ & $3.5 \pm 1.4$ \\
\hline
\end{tabular}

ROIs. ${ }^{18}$ The superior sagittal sinus is close to the skull and thus its image can be potentially degraded by beam-hardening artifacts when low kilovolt(peak) and milliampere-second settings are used. This phenomenon is more evident in the skull base or the higher convexity due to greater skull thickness. In our study, we did not find similar correlations in CBF and CBV maps. However, MTT maps seem to be the most susceptible of the 3 parameters to different factors in our and other studies. ${ }^{15,16,18} \mathrm{We}$ also found more oscillating time-attenuation curve waves and lower SNRs in the superior sagittal sinus when 80 -mAs tube current was used, but the difference between the standard and low mAs groups did not reach significance. Age is another factor that should be taken into consideration because osteosclerotic changes in the skull are more common in the aged population. Manual check of the proper position of VOF to avoid such influence of artifacts should be carefully performed in the setting of lower dose CTP.

IR algorithms are increasingly used in CT imaging due to continuous improvement in computer hardware and software. The advantage of this technique is its noise reduction. In general, the higher percentage of IR (the higher level of iDose ${ }^{4}$ ) with which the dataset is reconstructed, the higher SNR it achieves. Its major drawback is a smoothing effect near the boundaries, producing an "oil-painting" appearance. ${ }^{26,27}$ As a result, 100\% IR is not used in daily practice due to its unrecognized imaging texture. However, in CTP, the hemodynamic parameters rather than the regional tissue characteristics are the major concerns. Thus, this algorithm is increasingly being adopted to enhance the performance of lower dose CTP. ${ }^{28}$

The moderate correlations of the SNRs in CBV, CBF, and MTT were lower than expected, probably due to the region-ofinterest selection bias. Currently available software in our institution cannot provide automatic side-by-side region-of-interest comparison of 2 different perfusion datasets; thus, manual selection was required, which may have increased the intra- and interobserver variabilities. This effect was most profound in the arte- rial and venous ROIs due to relatively high noise caused by concentrated iodine, which led to different arterial input functions and thus changed the subsequent variables, including CBV, $\mathrm{CBF}$, and MTT.

Our case number was relatively small, and further investigation with a larger patient population is needed. Our sample consisted of patients undergoing post-carotid stent placement follow-up procedures. Two cases of restenosis were successfully confirmed by perfusion maps. However, clinical applications of lower dose CT in acute stroke with color-coded perfusion imaging deserve further validation. It should be feasible because the difference between normal and infarcted tissues is more evident than that between normal and oligemic tissue.

To our knowledge, this is the first study to explore the relationship between IR and perfusion parameters in CTP. In our initial experience, lower dose CTP can save up to $20 \%$ of the dose with the same perfusion parameters regardless of the reconstruction algorithm used. It will potentially enhance its applications in peritherapeutic monitoring of hemodynamic changes while treating neurovascular disorders. For example, it may be of use in selecting patients with cerebrovascular insults who will potentially benefit from thrombolytic therapy beyond current therapeutic windows or in predicting clinical outcome so as to tailor further management. ${ }^{29,30}$ Furthermore, the improved SNRs from the lower dose CTP dataset may diminish the noise of reformatted CTA in selected patients with acute ischemic insults. However, its accuracy in detecting aneurysms or other vascular anomalies deserves further investigation. ${ }^{31}$ The sequential hemodynamic information may further trim the performance of 3D-DSA by eliminating the unwanted venous structures. This may open a door to reducing the radiation dose for the comprehensive $\mathrm{CT}$ protocols in patients with neurovascular disorders. ${ }^{32}$ On the other hand, although IR algorithms increased the SNRs of axial imaging, they did not restore imaging quality in cases in which the original dataset was deteriorated by artifacts when further low milliampereseconds were attempted. Other methods to improve imaging quality should be explored.

\section{CONCLUSIONS}

Use of IR algorithms can achieve better SNRs in dynamic enhancing imaging with a $20 \%$ reduction in radiation dose and is a promising approach to improve the imaging quality of reformatted CTA. Meanwhile, it does not alter the absolute values or increase the SNRs in the CBF, CBV, and MTT color maps. The subjective 
imaging quality of CBF in lower dose CTP was slightly worse compared with that in standard CTP. Lower dose CTP can render peritherapeutic monitoring of hemodynamic changes more feasible for patients with neurovascular disorders. Further effort to lower the radiation dose should be pursued with other methods because IR cannot eliminate beam-hardening artifacts caused by skull and contrast due to the photon-starvation effect.

\section{REFERENCES}

1. Kalra MK, Prasad S, Saini S, et al. Clinical comparison of standarddose and $50 \%$ reduced-dose abdominal CT: effect on image quality. AJR Am J Roentgenol 2002;179:1101-06

2. Hara AK, Paden RG, Silva AC, et al. Iterative reconstruction technique for reducing body radiation dose at CT: feasibility study. $A J R$ Am J Roentgenol 2009;193:764-71

3. Kilic K, Erbas G, Guryildirim M, et al. Lowering the dose in head CT using adaptive statistical iterative reconstruction. AJNR Am J Neuroradiol 2011;32:1578-82

4. Kligerman S, Mehta D, Farnadesh M, et al. Use of a hybrid iterative reconstruction technique to reduce image noise and improve image quality in obese patients undergoing computed tomographic pulmonary angiography. J Thorac Imaging 2013;28:49-59

5. Rapalino O, Kamalian S, Kamalian S, et al. Cranial CT with adaptive statistical iterative reconstruction: improved image quality with concomitant radiation dose reduction. AJNR Am J Neuroradiol 2012;33:609-15

6. Korn A, Fenchel M, Bender B, et al. Iterative reconstruction in head $\mathrm{CT}$ : image quality of routine and low-dose protocols in comparison with standard filtered back-projection. AJNR Am J Neuroradiol 2012;33:218-24

7. Funama Y, Taguchi K, Utsunomiya D, et al. Combination of a lowtube-voltage technique with hybrid iterative reconstruction (iDose) algorithm at coronary computed tomographic angiography. J Comput Assist Tomogr 2011;35:480-85

8. Wintermark M, Lev MH. FDA investigates the safety of brain perfusion CT. AJNR Am J Neuroradiol 2010;31:2-3

9. Trojanowska A, Drop A, Jargiello T, et al. Changes in cerebral hemodynamics after carotid stenting: evaluation with CT perfusion studies. J Neuroradiol 2006;33:169-74

10. Waaijer A, van Leeuwen MS, van Osch MJ, et al. Changes in cerebral perfusion after revascularization of symptomatic carotid artery stenosis: CT measurement. Radiology 2007;245:541-48

11. Konstas AA, Wintermark M, Lev MH. CT perfusion imaging in acute stroke. Neuroimaging Clin N Am 2011;21:215-38

12. Mishra N, Albers G, Davis S, et al. Mismatch-based delayed thrombolysis: a meta-analysis. Stroke 2010;41:e25-e33

13. Abels B, Klotz E, Tomandl BF, et al. CT perfusion in acute ischemic stroke: a comparison of 2-second and 1-second temporal resolution. AJNR Am J Neuroradiol 2011;32:1632-39

14. Hirata M, Murase K, Sugawara $Y$, et al. A method for reducing radiation dose in cerebral CT perfusion study with variable scan schedule. Radiat Med 2005;23:162-69

15. Hom J, Dankbaar JW, Schneider T, et al. Optimal duration of acquisition for dynamic perfusion CT assessment of blood-brain barrier permeability using the Patlak model. AJNR Am J Neuroradiol 2009;30:1366-70

16. Wintermark M, Smith WS, Ko NU, et al. Dynamic perfusion CT: optimizing the temporal resolution and contrast volume for calculation of perfusion CT parameters in stroke patients. AJNR Am J Neuroradiol 2004;25:720-29

17. Wintermark M, Maeder $P$, Verdun FR, et al. Using $80 \mathrm{kV}$ p versus 120 $\mathrm{kVp}$ in perfusion CT measurement of regional cerebral blood flow. AJNR Am J Neuroradiol 2000;21:1881-84

18. Kealey SM, Loving VA, Delong DM, et al. User-defined vascular input function curves: influence on mean perfusion parameter values and signal-to-noise ratio. Radiology 2004;231:587-93

19. Greitz T. A radiologic study of the brain circulation by rapid serial angiography of the carotid artery. Acta Radiol Suppl 1956;140:1-123

20. Lin CJ, Hung SC, Guo WY, et al. Monitoring peri-therapeutic cerebral circulation time: a feasibility study using color-coded quantitative DSA in patients with steno-occlusive arterial disease. AJNR Am J Neuroradiol 2012;33:1685-90

21. Kudo K, Sasaki M, Ogasawara K, et al. Difference in tracer delayinduced effect among deconvolution algorithms in CT perfusion analysis: quantitative evaluation with digital phantoms 1. Radiology 2009;251:241-49

22. Kudo K, Sasaki M, Stergaard LO, et al. Susceptibility of Tmax to tracer delay on perfusion analysis: quantitative evaluation of various deconvolution algorithms using digital phantoms. J Cereb Blood Flow Metab 2011;31:908-12

23. Konstas AA, Goldmakher GV, Lee TY, et al. Theoretic basis and technical implementations of CT perfusion in acute ischemic stroke. Part 1. Theoretic basis. AJNR Am J Neuroradiol 2009;30:662-68

24. Waaijer A, van der Schaaf IC, Velthuis BK, et al. Reproducibility of quantitative $\mathrm{CT}$ brain perfusion measurements in patients with symptomatic unilateral carotid artery stenosis. AJNR Am J Neuroradiol 2007;28:927-32

25. Fiorella D, Heiserman J, Prenger E, et al. Assessment of the reproducibility of postprocessing dynamic CT perfusion data. AJNR Am J Neuroradiol 2004;25:97-107

26. Wang G, Snyder DL, O'Sullivan JA, et al. Iterative deblurring for CT metal artifact reduction. IEEE Trans Med Imaging 1996;15:657-64

27. McCollough CH, Primak AN, Braun N, et al. Strategies for reducing radiation dose in CT. Radiol Clin N Am 2009;47:27-40

28. Fardanesh M, Wang Q, Mehta D, et al. Evaluation of the impact of major dose reduction for quantitative brain perfusion studies using a low dose simulation algorithm. In: Proceedings of the Annual Meeting of Radiological Society of North America, Chicago, Illinois. November 27-December 2, 2011

29. Nogueira RG, Schwamm LH, Hirsch JA. Endovascular approaches to acute stroke. Part 1 . Drugs, devices, and data. AJNR Am J Neuroradiol 2009;30:649-61

30. Paciaroni M, Caso V, Agnelli G. The concept of ischemic penumbra in acute stroke and therapeutic opportunities. Eur Neurol 2009;61:321-30

31. Morhard D, Wirth CD, Fesl G, et al. Advantages of extended brain perfusion computed tomography. Invest Radiol 2010;45:363-69

32. Smith $A B$, Dillon WP, Gould R, et al. Radiation dose-reduction strategies for neuroradiology CT protocols. AJNR Am J Neuroradiol 2007;28:1628-32 